\section{The NEST Laboratory: The Art of a Multi-User Facility}

\author{
Scott Streiker and Rachel Smith \\ University of Dayton, Dayton, $\mathrm{OH}$ \\ Scott.Streiker@udri.udayton.edu
}

Radiolaria are marine protozoa that have thrived in the world's oceans for millions of years. They are particularly unique among marine plankton in that they build silica skeletons, which have allowed them to be preserved in the fossil record. These skeletons are ornate and complex and often demonstrate perfect geometric form and symmetry. The complex and beautiful glass-like structures are visually interesting when examined with electron microscopy. These attributes, coupled with their availability, size, ease of mounting and preparation make them superb specimens for introducing students to the use of electron microscopy (EM).

At the University of Dayton's Nanoscale Engineering Science and Technology (NEST) Laboratory, students are taught many aspects of electron microscopy. As a multi-user facility, and in order to ensure that all users can obtain the quality of data they need quickly and efficiently, it is of the utmost importance that all users become knowledgeable about the proper and safe operation of the instrument. The radiolaria tutorial serves as an excellent foundation to suit those needs.

Vials of fossil radiolaria can be purchased from several different suppliers of natural materials. The radiolaria are received as an aqueous suspension and are pre-washed, so no further preparation is required. Specimens are easily mounted onto carbon-tape covered aluminum stubs by placing a drop of the suspension on the tape and allowing the water to evaporate. Once dried, the sample surface is coated using a Denton Vacuum Desk II Cold Sputter unit to form a conductive gold layer. Sputtering for 30 seconds at 45 milliamps and 50 millitorr typically provides a coating of approximately 100 angstroms. Users are then instructed in basic operation of the instrument, such as loading, alignment, how to determine the ideal accelerating voltage, operation in both low magnification and high magnification modes.

The many unusual shapes and contours of the radiolaria present an excellent surface on which to learn image control techniques. Emphasis is placed on how to optimize brightness and contrast to
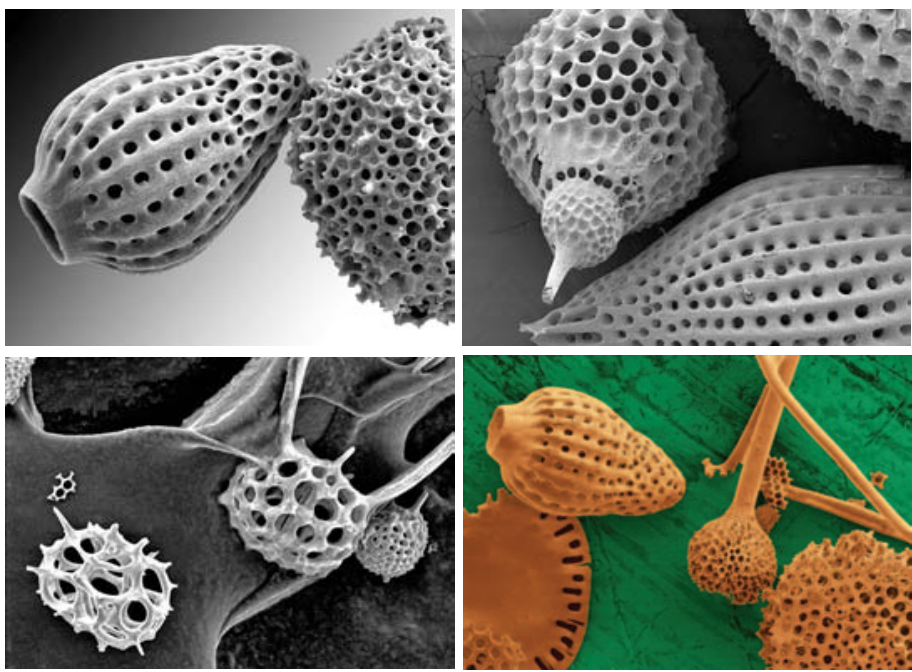

Figure 1: High Resolution SEM photomicrographs of radiolaria. Images were obtained using a Hitachi S-4800 HRSEM at X300 original magnification. ensure there is no loss of detail in the shadows and highlights, and how to stigmate the image to improve sharpness and clarity. After images have been optimized, further instruction details how to annotate, interpret and store the data.

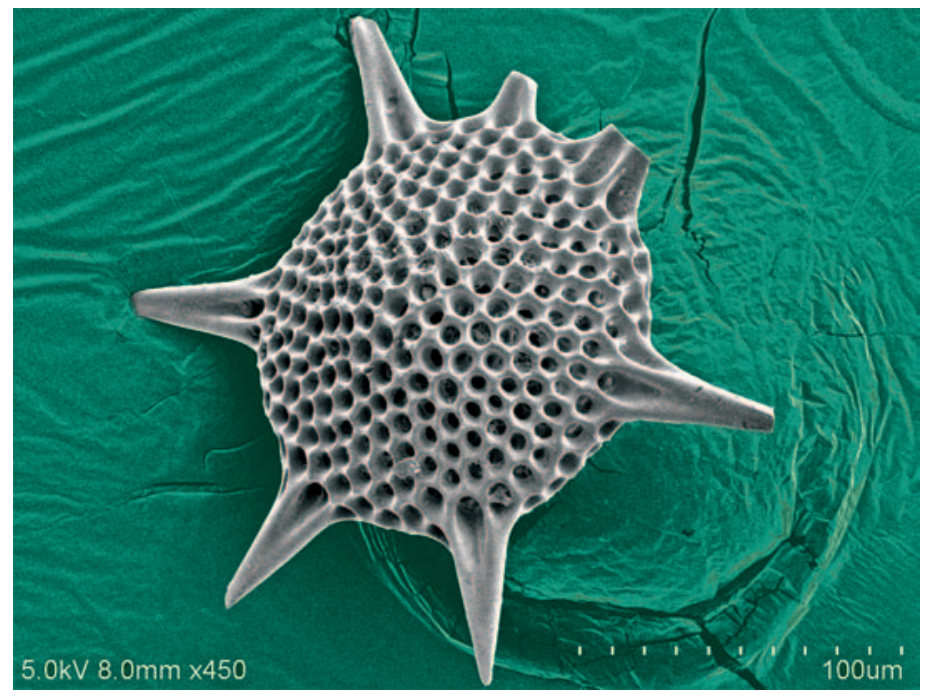

Figure 2: High Resolution SEM photomicrograph of a radiolarian obtained using a Hitachi S-4800 HRSEM at x450 original magnification.

The scanning electron microscope (SEM) photomicrographs, shown in figures 1 and 2 were captured under various magnifications using a Hitachi S-4800 High-Resolution SEM (HRSEM). Along with observation of the geometric features, the images in figure 2 clearly show arm-like spikes projecting from the main body, called axopods. These projections aid in maintaining buoyancy and capturing prey. For each sample, the working distance was $8 \mathrm{~mm}$, with an accelerating voltage of $5 \mathrm{kV}$ and a filament current of $10 \mu \mathrm{A}$.

The resulting images provide only a glance into the capabilities of the HRSEM. With sizes on the order of hundreds of microns, radiolaria are comparatively large samples. The HRSEM can be used to analyze virtually any non-volatile solid at an ultimate resolution of one nanometer $\left(1 \times 10^{-9} \mathrm{~m}\right)$ and is only one instrument among many that can be used for such analysis. The NEST laboratory hosts numerous instruments that can be used to image, characterize and quantify materials at the nanoscale.

The NEST laboratory was established in 2004 with funding from Ohio's Third Frontier Project, the Wright Brothers Institute and the University of Dayton (UD) and its Research Institute. It is a multi-user facility equipped with three electron microscopes, a micro-Raman spectrometer and a single-crystal $\mathrm{x}$-ray diffractometer, all of which allow the interrogation, characterization, manipulation and fabrication of materials and devices at the nanoscale. These capabilities have been used extensively in ongoing research at UD, primarily in the fields of materials science, biology and electrooptics. Currently, more than 80 UD faculty, postdoctoral fellows, graduate students and undergraduate students, UD Research Institute scientists, and researchers from local companies utilize one or more of the instruments in the NEST laboratory.

The electron microscopes in the NEST lab have been used to study a range of materials including nanoparticles such as carbon nanotubes, thin films for semi-conductors, optical arrays, and biological materials such as porcine vessels. The mission of the NEST $\mathrm{lab}$ is to enable and promote collaborative sponsored research, 


\section{It's Difficult To Do A Side-By-Side Comparison When Your Coater Stands Alone.}

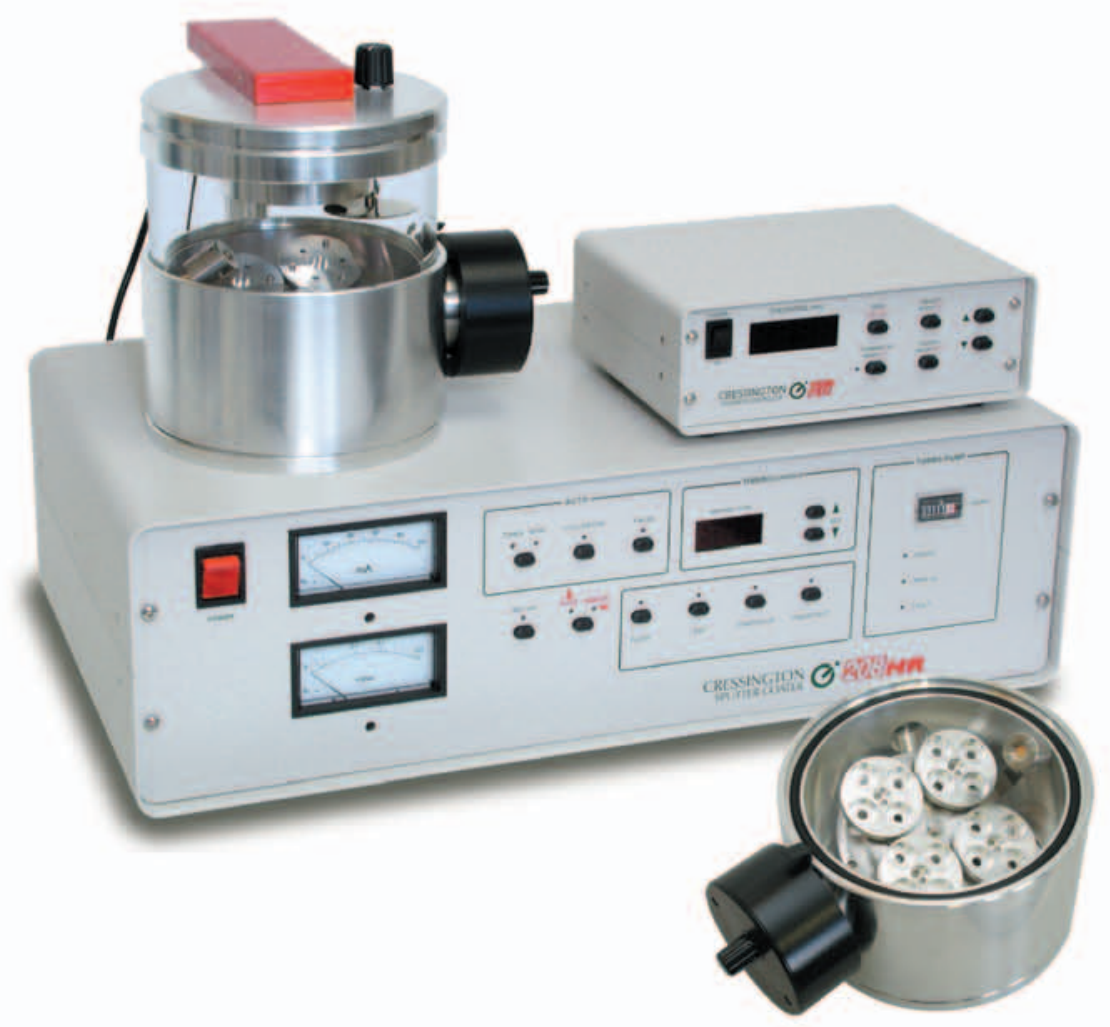

\section{High Resolution Sputter Coater 208HR for FE-SEM}

\section{Superior Features:}

- Wide Choice of Coating Materials

- Precision Thickness Control

- Multiple Sample Stage Movements

- Variable Chamber Geometry

- Wide Range of Operating Pressures

- Compact Modern Benchtop Design 
provide technical support to various research and development programs, facilitate the instruction of laboratory classes related to nanotechnology and serve as a resource to industry to facilitate nanotechnology transition and commercialization. Radiolaria are just one of the many teaching tools used to realize these goals-the first stepping stones towards imaging smaller and even more complex materials.
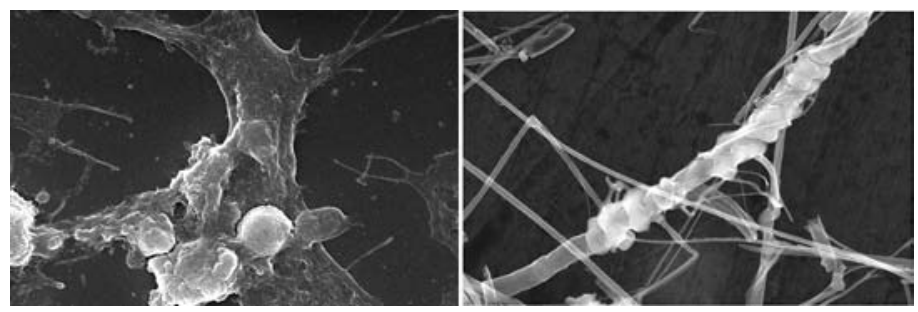

Figure 3: High Resolution SEM photomicrographs of oyster hemocytes (left) and carbon nanotubes (right). The oyster hemocyte image was obtained at x6,000 original magnification. The carbon nanotubes were obtained at $\times 2,500$ original magnification.

The tools of microscopy are becoming even more important in the life sciences and related disciplines. Several researchers from the UD Department of Biology are using the NEST facility to examine the physical, anatomical, cellular, subcellular features of living organisms, tissues, and/or cells to address a variety of questions in biology, biomedicine, and environmental biology. Ongoing projects include the evaluation of mammalian blood vessel architecture using ESEM, the characterization of pili on mutants of Pseudomonas aerugenosa using TEM, subcellular localization of cytoplasmic proteins in mammalian cells by confocal microscopy, and characteristics of the zona pellucida of canine oocytes using HREM.

A group of scientists from the University of Dayton Research Institute in collaboration with researchers at Clemson University are utilizing some of the instruments at the NEST laboratory to

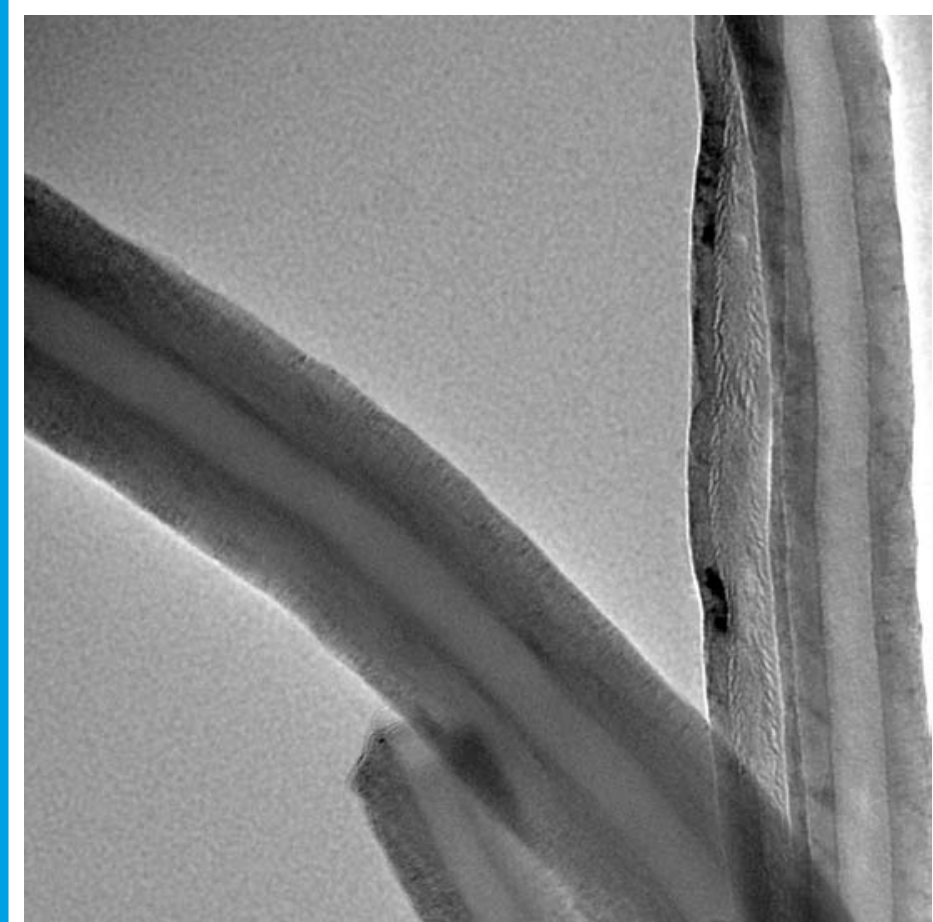

Figure 4: Transmission Electron Microscope (TEM) photomicrograph of a carbon nanotubes. The image was obtained using a Hitachi H-7600 TEM with an accelerating voltage of $60 \mathrm{kV}$. better understand the formation of natural minerals through their study of the biomineralization mechanism of the Eastern oyster. Marine and freshwater mollusks produce elegant shells made of calcium carbonate, many of which are prized by collectors. Shell of the Eastern oyster, Crassostrea virginica, is a lightweight yet strong natural ceramic which has both mineral and organic phases. Calcium carbonate crystal formation at the growing shell margin is initiated by oyster hemocytes (blood cells) and can be seen in figure 3.

One of the fields of study that receives the most attention at the NEST is carbon materials such as carbon nanotubes (CNTs) and carbon fibers. With an ultimate resolution of $1 \mathrm{~nm}$, the HRSEM is especially well-suited to imaging CNT's, which can have diameters on the order of a few tens of nanometers. Figure 3 shows an image of a carbon nanotube.

The other major characterization equipment in the NEST lab includes a transmission electron microscope (TEM) and an environmental scanning electron microscope (ESEM). The TEM, has an ultimate resolution of 0.2 nanometers which makes it ideal for imaging carbon nanotubes, as can be seen in figure 4. The ESEM can be operated with air or water vapor in the sample chamber while maintaining a high vacuum on the electron gun, allowing the analysis of wet or non-conductive samples. The ESEM is frequently used for biologic samples and is a useful tool for identifying features such as form and structure of organisms. The nanocharacterization facility also features an ultramicrotome, X-ray diffractometer, surface profiler, micro-Raman spectrometer, confocal microscope, inverse gas chromatograph and BET surface area analyzer.

The X-ray diffractometer housed in the NEST laboratory is Oxford Diffraction Xcalibur3 diffractometer system, equipped with an Enhance-X-ray source capable of producing either a very intense $(2 \mathrm{~kW}) \mathrm{Cu}$ or Mo X-ray beam. The diffractometer is equipped with a Cryojet system to allow for data collection at temperatures down to $100 \mathrm{~K}$. The system is ideal for the analysis of single crystals and has also been used to examine fiber specimens and powder samples. Much of the work carried out to date has involved establishing a strategy for the collection of precise X-ray intensity data on single crystals at low temperature. The diffraction data is then used to determine crystal structures including bond lengths and bond angles.

The NEST laboratory's Renishaw inVia Reflex micro-Raman spectrometer has two lasers to provide excitation wavelengths of $785 \mathrm{~nm}$ and $514 \mathrm{~nm}$. The laser spot size can be varied from $1 \mu \mathrm{m}$ to $300 \mu \mathrm{m}$, allowing for examination of very small regions of the sample. Spectra can be obtained using a variety of acquisition modes such as single point scan, line mapping and area mapping. It is capable of making confocal measurements to create depth profiles of samples with $2.5 \mu \mathrm{m}$ resolution. It is used for a wide variety of applications, ranging from chemical analysis of carbon nanotubes to stress measurements in multilayer structures.

The staff at the NEST laboratory includes Scott Streiker, who is responsible for electron microscopy (TEM, SEM) analysis and training of new users, and Rachel Smith, whose expertise includes Raman microscopy, surface profilometry and various scanning probe microscopy techniques.

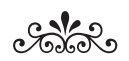




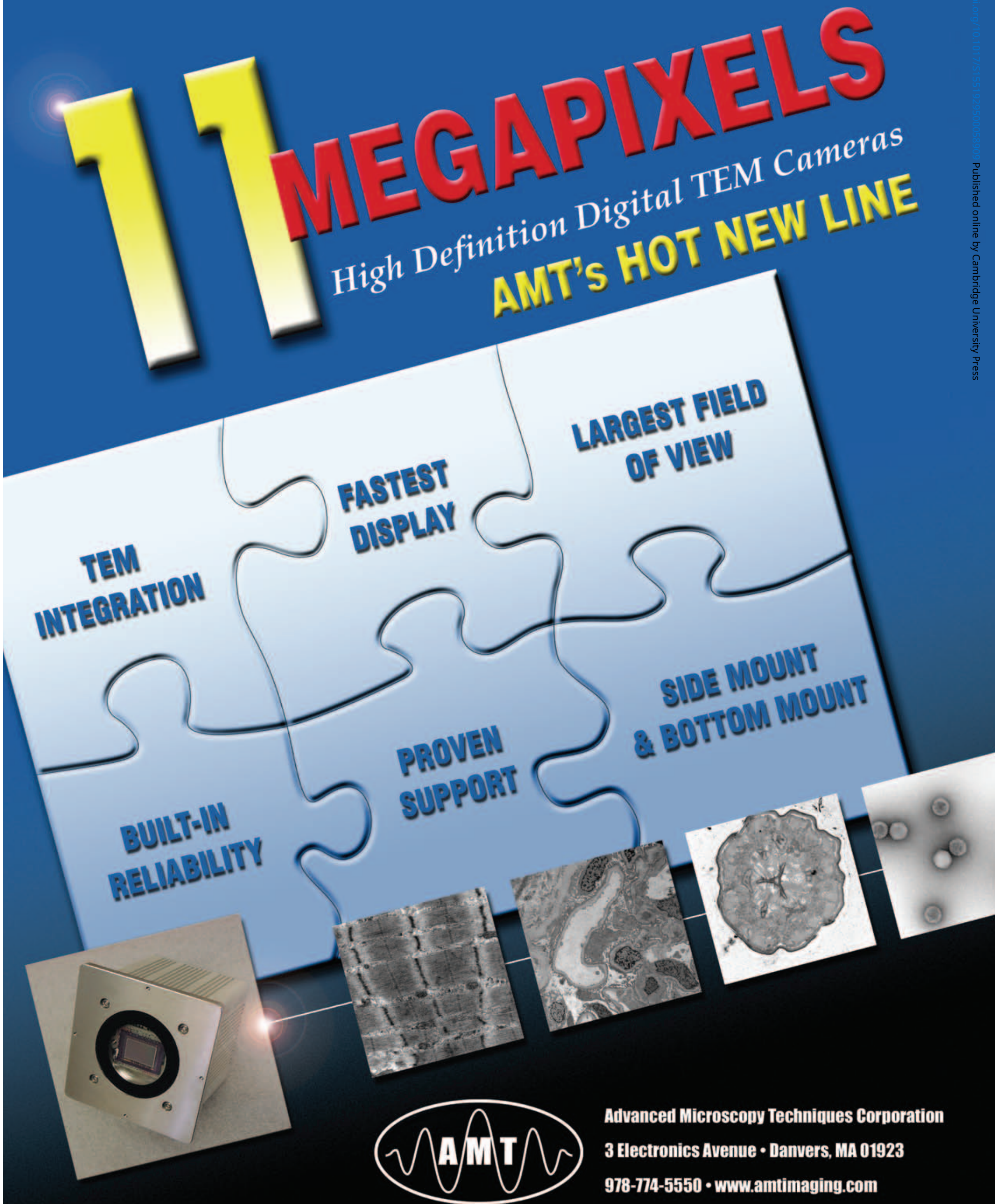

